\title{
What Changes in Poly(3-Hydroxybutyrate) (PHB) When Processed as Electrospun Nanofibers or Thermo-Compression Molded Film?
}

\author{
Artur Caron Mottin ${ }^{a, b}, *$, Eliane Ayres ${ }^{a, b}$, Rodrigo Lambert Oréfice ${ }^{c}$, Jairo José Drummond Câmara ${ }^{a, b}$ \\ ${ }^{a}$ Thematic Network in Materials Engineering - REDEMAT, School of Mining Engineering, \\ Ouro Preto, $M G$, Brazil. \\ ${ }^{b}$ School of Design, Department of Materials, Technologies and Processes, Minas Gerais State University - UEMG, \\ Belo Horizonte, MG, Brazil. \\ ${ }^{c}$ Department of Metallurgical and Materials Engineering, Federal University of Minas Gerais - UFMG, \\ Pampulha, Belo Horizonte, MG, Brazil.
}

Received: May 18, 2015; Revised: August 20, 2015; Accepted: November 20, 2015

In the present study, PHB film was prepared by thermo-compression molding and PHB nanofibers mat was produced through electrospinning process. The nanofibers morphology was confirmed by SEM. The film and nanofibers crystallinity degree was estimated by different methods. FTIR and WAXD indicated that PHB film is more crystalline than PHB nanofibers. However, DSC analysis suggested that a PHB nanofibers phase change might occur while the temperature rises. SAXS indicated that PHB film and nanofibers have low electron density contrast between the alternating crystalline and amorphous layers. The influence of nanofiber morphology on the mechanical, surface and thermal properties was also shown in TG analysis, tensile tests and contact angle measurements.

Keywords: PHB; Electrospinning; Thermo-compression molded film; Crystallinity degree.

\section{Introduction}

Bacterial polyhydroxyalkanoates (PHAs) are the unique family of polymers that acts as a carbon/energy store for more than 300 species of Gram-positive and Gram-negative bacteria as well as a wide range of archaea ${ }^{1}$.

The French bacteriologist Lemoigne was the first to isolate and characterize poly-3-hydroxybutyrate (PHB) from bacteria between 1923 and 1927, and showed that this extract could be cast in to a transparent film and, most importantly, these biopolymers were completely biodegradable ${ }^{1}$. $\mathrm{PHB}$ is an eco-friendly alternative for several applications, for example, PHB have demonstrated great potential as a material for food packaging ${ }^{2}$. Also in the area of biomaterials the osteogenic potential of pure PHB patches has been evaluated by Gredes et al. ${ }^{3}$ These authors concluded that the tested PHB patches featured a good biocompatibility and an osteoconductive character.

In vivo $\mathrm{PHB}$ granules are fully amorphous, but during recovery, the amorphous granules environment changes and may cause the crystallization of the particles ${ }^{4}$. When pure PHB is heated above its melting point $\left(\sim 180^{\circ} \mathrm{C}\right)$, it becomes a fully amorphous melt. Upon cooling, the PHB molecules crystallize, forming antiparallel helical chains linked by $\mathrm{C}$ $-\mathrm{H} \cdots \mathrm{O}$ hydrogen bonds between the carbonyl $(\mathrm{C}=\mathrm{O})$ and methyl $\left(\mathrm{CH}_{3}\right)$ groups of the polyester backbone ${ }^{4}$. The final PHB polymer properties depend on the crystallization degree, which depends on the PHB processing conditions. So, it is very useful to characterize the PHB crystallization and properties under different processing methods.

*e-mail address: artur.mottin@uemg.br
In an electrospinning process, a strong electrostatic field is applied to a polymer solution held in a syringe and feed through a needle. The fiber jet travels through the atmosphere allowing the solvent to evaporate, thus leading to the solid polymer fibers deposition on the collector ${ }^{5}$. Blends of several ratios of poly(3-hydroxybutyrate-cohydroxyvalerate) (PHBV) and chitosan were electrospun to produce fibrous membranes ${ }^{6}$. In this case, the goal was to combine these two biocompatible and biodegradable polymers, thus creating a new material with potential biomedical application. Another example is the PHB (core) and gelatin (sheath) coaxial electrospinning, producing porous fibrous mats with potential as a scaffold (dermal substitute) for skin regeneration ${ }^{7}$.

The present work, thus, aims to show the possible PHB properties changes, mainly in the crystallinity, when this biopolymer was processed through thermo compression molding or electrospinning.

\section{Experimental}

\subsection{Materials}

Poly(hydroxybutyrate) (PHB) (Biocycle 1000, $\mathrm{Mw}=600,000 \mathrm{~g} \mathrm{~mol}^{-1}$ ) was provided by PHB Industrial S.A (Serrana, Brazil). According to the manufacturer, the product has the properties shown in Table 1. Chloroform and N, N - dimethylformamide (DMF) were supplied by Synth (Brazil). All reagents were used as received without any pre-treatment. 
Table 1. ASTM properties of Biocycle 1000

\begin{tabular}{lc}
\hline \multicolumn{3}{c}{ Physical properties } \\
\hline $\begin{array}{l}\text { Density } \\
\text { Melt flow index }\end{array}$ & $1.20 \mathrm{~g} \mathrm{~cm}^{-3}$ \\
\hline \multicolumn{2}{c}{ Mechanical properties } \\
\hline Tensile strength & $32 \mathrm{~g} / 10 \mathrm{~min}$ \\
Tensile elongation at break & $4 \%$ \\
Flexural modulus & $2,200 \mathrm{MPa}$ \\
Izod notched impact strength & $28 \mathrm{Jm}^{-1}$ \\
\hline \multicolumn{2}{c}{ Thermal properties } \\
\hline Melting point & $170-175^{\circ} \mathrm{C}$ \\
Heat distortion temperature & $65^{\circ} \mathrm{C}$ \\
Vicat softening temperature & $135^{\circ} \mathrm{C}$ \\
\hline
\end{tabular}

\subsection{PHB thermo-compression molded film preparation}

PHB film was prepared by thermo-compression molding using a SAGEC manual hydraulic press with heated platens, manual thermostat control and maximum pressure of $30,000 \mathrm{psi}$. First the press was pre-heated at $170{ }^{\circ} \mathrm{C}$ and enough PHB powder was placed in a stainless steel frame with top and bottom covers $(200 \times 200 \times 1.2 \mathrm{~mm})$. Films were obtained at $4000 \mathrm{psi}$ of pressure during $2 \mathrm{~min}$. For successful demolding, Kapton $^{\circledast}$, a heat sealable grade polyimide film produced by DuPont, was used as anti-adhesion layer.

\subsection{PHB Electrospinning}

First, PHB was dissolved in a chloroform and DMF mixture at a 9:1 ratio to yield a homogeneous solution with $6 \%$ wt concentration. The solubilization was carried out in a magnetic stirrer (TECNAL, model 0851) for 3 hours at $50{ }^{\circ} \mathrm{C}$. The electrospinning apparatus was an equipment manufactured by INSTOR (Porto Alegre, Brazil), that makes use of a $5 \mathrm{~mL}$ syringe (Artiglass, Italy) connected to a stainless steel needle $(0.7 \mathrm{~mm}$ in diameter $)$, with a polarity reversible high voltage power supply (Gamma High Voltage Research, USA) with one electrode directly clamped onto the metal needle and the other electrode clamped onto a rotating collector of low rotational speed (40 rpm). During the electrospinning process, the solution was ejected from the positively charged needle $(11 \mathrm{kV})$ tip by using a syringe pump, connected to the syringe through a Teflon ${ }^{\circledR}$ tube, used to push the syringe to deliver a constant and stabilized polymer solution mass flow. The fibers were randomly deposited onto the collector surface connected to ground with a zero polarity. The distance between the needle tip and the collector was of $10 \mathrm{~cm}$. The experiment was conducted at $25^{\circ} \mathrm{C}$ and $50 \%$ of relative humidity. The aforementioned process conditions were previously determined by extensive experimental work.

\subsection{Characterization of PHB films and nanofibers mat}

\subsubsection{Scanning electron microscopy (SEM)}

The PHB nanofibers mat morphology was analyzed via scanning electron microscopy (SEM) (Shimadzu model Vega $3 \mathrm{LM}$ ) at an accelerating voltage of $15 \mathrm{kV}$. Samples for SEM were coated with gold using a sputter coater (SPI Sputter Coater, SPI Supplies, PA, USA). To determine the nanofibers diameter and the diameters distribution, the nonwoven mat was analyzed using 100 individual measurements, which were obtained from the SEM micrograph by image analysis software (Image J, National Institutes of Health, USA).

\subsubsection{Fourier transform infrared spectroscopy (FTIR)}

Fourier transform infrared (FTIR) experiments were performed with the aid of a Thermo Scientific Nicolet ${ }^{\mathrm{TM}}$ 6700 FT-IR spectrometer at a $4 \mathrm{~cm}^{-1}$ resolution and 256 co-added scans. The spectra were obtained with dry films using the ATR (attenuated total reflection) technique, in which the samples were pressed against a $\mathrm{ZnSe}$ crystal and spectra were collected from $650-4000 \mathrm{~cm}^{-1}$.

\subsubsection{X-ray diffraction (XRD)}

The crystallographic structure of PHB different morphologies (film and nanofibers) was verified using an X-ray diffractometer (Shimadzu, model XRD-7000) equipped with a $\mathrm{Cu}$ tube $(\mathrm{Cu} K \alpha$ radiation, $\lambda=1.54 \AA)$ operating at $40 \mathrm{kV}$ and $30 \mathrm{~mA}$. The samples were scanned at $2^{0} \mathrm{~min}^{-1}$ between $2 \theta=5$ and $80^{\circ}$.

\subsubsection{Mechanical properties}

PHB film and nanofibers mat were characterized in their tensile properties, such as elastic modulus, tensile strength and elongation at break, using an Instron testing machine (model EMIC 3000) with load cell of $200 \mathrm{~N}$. Tensile properties were characterized according to ASTM D-638 and at least five specimens were tested to obtain the average values. A crosshead speed of $10 \mathrm{~mm} \mathrm{~min}^{-1}$ and a gage length of $13 \mathrm{~mm}$ were used for the tests.

\subsubsection{Differential scanning calorimetry (DSC)}

Differential scanning calorimetry (DSC) experiments were carried out using a Seiko-SII Nanotechnology Inc. model Exstar 7200 equipment. Samples (ca. 7 mg) were sealed in aluminum pans and measurements were performed in nitrogen atmosphere (flow rate of $40 \mathrm{~cm}^{3} \mathrm{~min}^{-1}$ ). First, samples were previously cooled at $-15^{\circ} \mathrm{C}$ and heated at $190^{\circ} \mathrm{C}$ with a $10^{\circ} \mathrm{C} \mathrm{min}^{-1}$ heating rate (first heating). Then samples were cooled down at $-15^{\circ} \mathrm{C}$ and submitted to heating until $190{ }^{\circ} \mathrm{C}$ (second heating).

\subsubsection{Small angle X-ray scattering (SAXS)}

To investigate the crystalline structure developed in the PHB different morphologies (film and nanofibers), two-dimensional (2-D) small-angle X-ray scattering (SAXS) measurements of synchrotron SAXS were performed using the beam line of the National Synchrotron Light Laboratory (LNLS, Campinas, Brazil). The high intensity and good collimation of the beam line have made it feasible to study the polymeric materials structural evolution through SAXS. A monochromatic beam of $1.55 \AA$ wavelength was used and the exposure time was $300 \mathrm{~s}$. The scattering intensity was registered using a Pilatus detector $(300 \mathrm{~K}, 84 \times 107 \mathrm{~mm})$ for SAXS with a sample to detector distance of $1976.5 \mathrm{~mm}$. 


\subsubsection{Thermogravimetric analysis (TG)}

The thermogravimetric analyses were performed with small dry samples weighting around $7 \mathrm{mg}$ using a Seiko-SII Nanotechnology Inc. model Exstar 7200 equipment under nitrogen atmosphere (flow rate of $30 \mathrm{~cm}^{3} \mathrm{~min}^{-1}$ ). Samples were heated from $25^{\circ} \mathrm{C}$ to $300^{\circ} \mathrm{C}$ at a $10^{\circ} \mathrm{C} \mathrm{min}^{-1}$ heating rate.

\subsubsection{Contact angle measurement}

Contact angle measurements were performed using the sessile drop technique with DIGIDROP goniometer from GBX instruments. The system is equipped with a CCD camera connected to a computer and to an automatic liquid dispenser. The contact angle was determined by placing a $10 \mu \mathrm{L}$ drop of water on the film or nanofiber mat surface using a syringe and images were immediately sent via the CCD camera to the computer for analysis. The results represent an average angle between the right and left angles. Three consecutive measurements were made at room temperature, using the Surface Energy software mode, which allows direct measurement of contact angle (in degrees).

\section{Results and discussion}

Electrospun mats of PHB fibers without beads were successful fabricated under the experimental conditions. With regards to the fibers size, the average diameter is in nanometer scale as shown in Figure 1.

Initial investigations with FTIR were concerned in detect any PHB crystalline morphology change occurred after processing viz. thermo-compression molding and electrospinning. FTIR spectra of pure as-received PHB powder, PHB film and PHB nanofibers mat are shown in Figure 2, in which no pronounced differences between the three spectra were observed.

Suitable infrared absorption bands can be used to study crystallinity in polymers. The infrared absorption spectra of the same polymer in the crystalline and amorphous states can differ for at least two reasons. First, specific intermolecular interactions may exist in the crystalline polymer, which lead to sharpening or splitting of certain bands; and second, some specific conformations may exist in one but not in the other phase, leading to bands which are characteristic exclusively of either crystalline or amorphous material ${ }^{8}$.

FTIR has been used by many authors to estimate the PHB crystallinity. Porter et al. ${ }^{4}$, for example, monitored the pure PHB crystallinity through an index (AI) calculated by the ratio between the bands at $1184 \mathrm{~cm}^{-1}$ and $1382 \mathrm{~cm}^{-1}$ intensity. According to them, when PHB is cooled from an amorphous melt at $180^{\circ} \mathrm{C}$ to a semi-crystalline solid at room temperature, the absorption intensity at $1184 \mathrm{~cm}^{-1}$ decreases as a function of time while the absorption intensity at $1382 \mathrm{~cm}^{-1}$ does not change during and can be taken as the reference. On the other hand, Xu et al., ${ }^{9}$ found that the band at $1380 \mathrm{~cm}^{-1}$ changed due to crystallization of polyhydroxyalkanoates. They have preferred another index, defined as the absorbance ratio of the crystalline band at $1230 \mathrm{~cm}^{-1}$ to the unchanged reference at $1453 \mathrm{~cm}^{-1}$. The band at $1230 \mathrm{~cm}^{-1}$ was proposed as the helical chains conformational band, since no amorphous bands of the same group could be found ${ }^{9}$.
The PHB crystallization degree is most commonly investigated by monitoring the $\mathrm{C}=\mathrm{O}$ stretching vibrational region $^{10-12}$. For this purpose, deconvolution of the $\mathrm{C}=\mathrm{O}$ stretching region was performed using a Gaussian-Lorentzian sum $\left(R^{2}=0.995\right)$. The bands found and their corresponding percent areas are presented in Table 2.

Zhang et al..$^{10}$, during their experiments with the PHB melt-crystallization process, observed that the intensity of the band at $1722 \mathrm{~cm}^{-1}$ increased gradually as a function of time while the band at $1743 \mathrm{~cm}^{-1}$ decreased during the crystallization process. In reason of this fact, they assigned the $1722 \mathrm{~cm}^{-1}$ band to the $v(\mathrm{C}=\mathrm{O})$ for the crystalline phase and $1743 \mathrm{~cm}^{-1}$ band to the $v(\mathrm{C}=\mathrm{O})$ for the amorphous phase. Wang et al. ${ }^{11}$ also assigned peaks at $1721 \mathrm{~cm}^{-1}$ and $1735 / 1745 \mathrm{~cm}^{-1}$ to the carbonyl stretch band in crystalline and amorphous phases respectively in the FTIR spectra of PHB electrospun nanofibers.

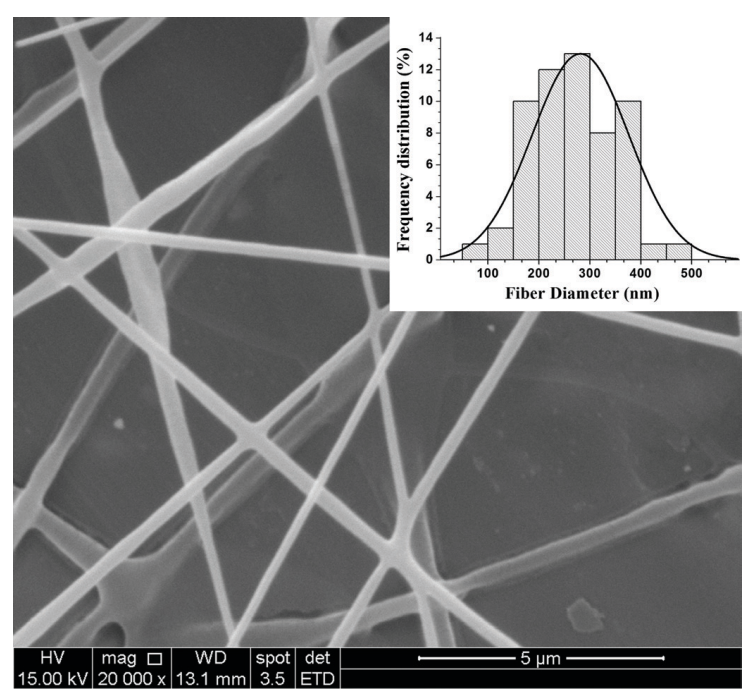

Figure 1. SEM micrograph of electrospun PHB nanofibers mat. (Magnification 20,000 X).

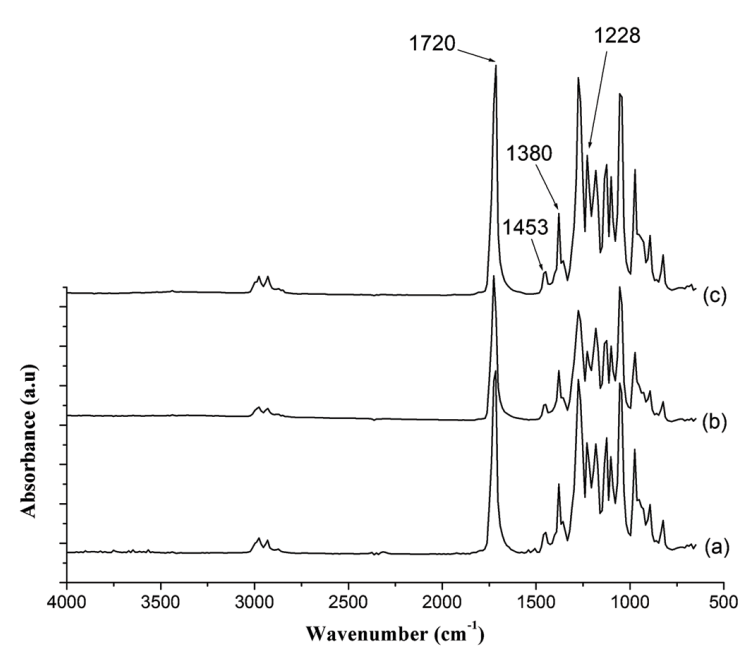

Figure 2. FTIR spectra of: (a) as-received PHB powder, (b) electrospun PHB nanofibers mat and (c) PHB thermo-compression molded film. 
Table 2. FTIR peaks and their corresponding areas after deconvolution of carbonyl region.

\begin{tabular}{clcl}
\hline Bands (area) & Powder & Film & Nanofibers \\
\hline 1 & $1686(1.48 \%)$ & $1686(6.39 \%)$ & $1687(4.34 \%)$ \\
2 & $1704(11.85 \%)$ & $1714(23.68 \%)$ & $1704(18.53 \%)$ \\
3 & $1720(33.03 \%)$ & $1719(41.97 \%)$ & $1720(22.90 \%)$ \\
4 & $1725(44.26 \%)$ & $1730(20.04 \%)$ & $1726(35.62 \%)$ \\
5 & $1744(9.39 \%)$ & $1745(7.92 \%)$ & $1742(18.61 \%)$ \\
\hline
\end{tabular}

The band at around $1730 \mathrm{~cm}^{-1}$ has been also ascribed as related to the crystalline part with a less ordered structure, but the assignment of such band is not clear ${ }^{10}$. The peak that appears at $1686 \mathrm{~cm}^{-1}$ has been described as a crystalline sensitive band and has been suggested to be related to the crystal defect caused by the interaction of an $\mathrm{OH}$ end group and $\mathrm{a}=\mathrm{O}$ group of $\mathrm{PHB}^{10}$. The assignment on the $1704 \mathrm{~cm}^{-1}$ or $1714 \mathrm{~cm}^{-1}$ band was not found in the literature, but is reasonable to speculate that this range of absorption is related to regions of well-packed chains with strong hydrogen bonds.

According to Table 2, PHB nanofibers mat presented the larger area of the band related to the amorphous phase (around $1745 \mathrm{~cm}^{-1}$ ). One possible theory is that, although they were collected onto a rotating collector, the speed was not sufficient to stretch the fibers. For this reason, they were randomly deposited giving rise to low crystallinity fibers.

This is not in agreement with the result reported by Pachekosky et al. ${ }^{12}$. Based on the investigation of FTIR carbonyl region, they found that processing conditions caused no effect in the crystallization degree of extruded PHB film. However, it is worth noting that such authors did not separate the carbonyl bands. Wang et al. ${ }^{11}$, on the other hand, concluded that the ratio of $\mathrm{A}_{1228} / \mathrm{A}_{1453}$ seems to be more appropriate to represent the PHB fibers relative crystallinity due the absence of required bands deconvolution. In this context, the indexes calculated through the ratio $\mathrm{A}_{1228} / \mathrm{A}_{1453}$ are shown in Table 3.

The indexes found are within the range of 2.17 and $2.08 / 2.40$ reported in the literature for PHB films and nanofibers respectively ${ }^{9,11}$. PHB film presented the highest crystallinity index (Table 3 ) and the lowest percentage area of amorphous peak (Table 2). However, in the case of PHB powder or nanofibers, the indexes found did not match with the percent areas of Table 2. Possibly this is due to the difficult deconvolution of the heavily overlapping peaks in carbonyl region.

To confirm the influence of the processing type on the PHB crystallinity, the thermo-compression molded film WAXD profile was compared with that of the electrospun nanofibers mat and both are shown in Figure 3.

The profiles related to the film and nanofibers presented accentuated differences. As previously shown through FTIR, the film was suggested to be more crystalline. In the film profile, two strong scattering intensity peaks were detected at around $2 \theta=13^{\circ}$ and $17^{\circ}$ assigned to be (020) and (110) of orthorhombic unit cell respectively ${ }^{13,14}$. Weaker reflections located at around $21.5^{\circ}$ and $22.5^{\circ}$ corresponding to (101) and (111) reflections of $\alpha$ PHB crystal were also detected ${ }^{14}$. Others reflections that were detected at around $26^{\circ}$ and $27^{0}$ are reported in literature as (130) and (040) respectively ${ }^{11}$.
Table 3. Absorption indexes (AI) calculated through the ratio $\mathrm{A}_{1228} / \mathrm{A}_{1453}$.

\begin{tabular}{lc}
\hline \multicolumn{1}{c}{ PHB } & AI $\left(\mathbf{A}_{\mathbf{1 2 2 8}} / \mathbf{A}_{\mathbf{1 4 5 3}}\right)$ \\
\hline PHB powder & 2.08 \\
Film & 2.44 \\
Nanofibers mat & 2.10 \\
\hline
\end{tabular}

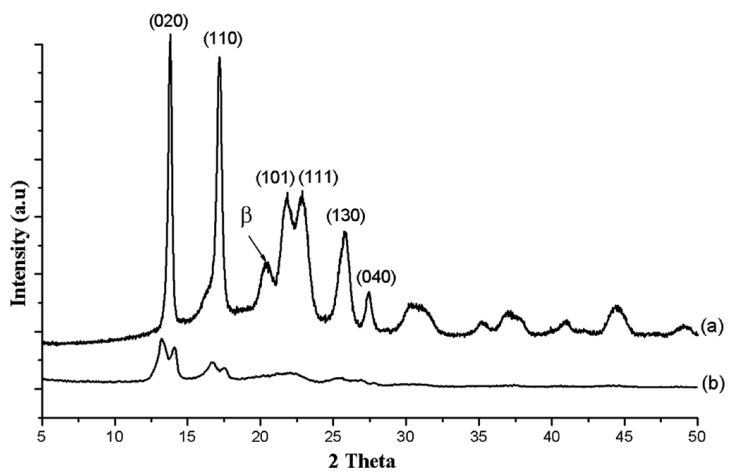

Figure 3. WAXD intensity profiles of PHB in: (a) thermo-compression molded film and (b) electrospun nanofibers mat. The diffraction planes associated with $\alpha$ crystalline form are indexed. The peak at around $2 \theta=200$ indicates the presence of $\beta$ crystalline form.

At around $2 \theta=20^{\circ}$ one can perceive a diffraction peak attributed to $\beta$ form crystals ${ }^{11}$.

In terms of crystals structures of PHB, the most common PHB molecule crystal structure is the $\alpha$-form, which is produced under the typical conditions of melt, cold or solution crystallization ${ }^{1}$. According to Laycock et al. ${ }^{1}$, the $\beta$-form of the PHB crystal, which can be obtained for uniaxially stretched films, is metastable and can be annealed back to the $\alpha$-form at $130{ }^{\circ} \mathrm{C}$ with a resulting increase in crystallinity. Taking this into account, we might assume that some stretching degree occurred during the film processing, causing an increase in crystallinity. Nevertheless this result is not usual to be found in literature. Pinto et al. ${ }^{15}$, for example, observed a decrease in the crystallinity due to the pressure application during PHB thermo-compression molding.

In the case of nanofibers, unlike the result reported by Wang et al. ${ }^{11}$, the reflection corresponding to $\beta$-form could not be perceived. The WAXD profiles showed by these authors revealed that PHB electrospun fibers possessed a small amount of $\beta$ form crystals with zigzag conformation. According to them, in the electrospinning process, the interlaced chains between the crystalline lamellae are further elongated during jet whipping leading to the development 
of the $\beta$-form. Likewise that in FTIR experiments, we might suppose that the speed of the rotating collector was not sufficient to stretch the fibers thereby preventing the development of $\beta$-form.

From the XRD patterns, the apparent crystal size $\mathrm{D}_{(020)}$ of PHB in the direction perpendicular to the 020 crystallographic plane was estimated by using Scherrer's equation ${ }^{16}$.

$D=\frac{k \lambda}{\beta \cos \theta}$

Where $\mathrm{k}$ is proportionality constant, $\lambda$ is the $\mathrm{X}$-ray wavelength (nm) and $\beta$ is the enlargement of the measured diffraction line at mid-height of its maximum intensity (in radian unit), and $\theta$ is the XRD peak position. The proportionality constant $\mathrm{k}$ is a function of the geometrical shape of the crystal. In the case of not knowing the geometry of the crystallites, it is assumed a spherical geometry with $\mathrm{k}$ value of 0.9 .

With this approach, the estimated average sizes of the crystals were found to be $266 \AA$ and $190 \AA$ for the film and electrospun nanofibers respectively and are consistent with the XRD patterns presented. Canetti et al. ${ }^{16}$ found larger crystal sizes (020) between $345 \AA$ and $790 \AA$ (setting the value of $\mathrm{k}$ equal to unit) for the thin films of PHB prepared by the solution-casting technique. As smaller the size of crystallites is, the broader diffraction peaks would be. Sometimes is difficult to recognize them as diffraction peaks. Taking this into account, the sizes of $\alpha$-form crystals found through Scherrer's equation are compatible with the diffractograms shown in Figure 4.

The thermal transitions of PHB thermo-compression molded film and nanofibers mat were determined by differential scanning calorimetry (DSC). The cooling and second heating are shown in Figure 4.

As can be seen in Figure 4, the curves look similar and the most remarkable event is the double melting peak presented in the heating curve for the two PHB forms. Fabri et al., ${ }^{17}$ reported that most of the bimodal melting profiles are due to the concurrent phenomena of melting and recrystallization followed by final melting at higher temperatures. According to them, this process is consequence of the crystals size distribution, and the melting temperature is directly proportional to the crystals size. Erceg et al., ${ }^{18}$ reported the appearance of a small shoulder before the maximum peak for PHB compression molded film without plasticizer when annealed at $190{ }^{\circ} \mathrm{C}$ and higher temperatures. They attributed this behavior to bimodal distribution of crystallite size resulting from changes in molecular weight due to random scission of long PHB chains, i.e. to the decrease in the molecular weight.

For polymers that melt and recrystallize during heating, like PHB, the melting temperature of original crystals can be obtained by operating at high crystallization temperatures and high heating rates ${ }^{19}$.

Other interpretation of the multiple melting has been given by Bezerra et al. ${ }^{20}$. According to them the two PA6 melting temperature peaks can be assigned to the two crystal distinct forms known in the literature as $\alpha$ and $\gamma$. In view of this, one might extrapolate to the PHB assigning the two melting temperature peaks to $\alpha$ and $\beta$ crystal forms.

The crystallinity $(\chi)$ was evaluated by comparing the area of the melting peak $\left(\Delta \mathrm{H}_{\mathrm{m}}\right)$ with the melting enthalpy of a $100 \%$ crystalline material $\left(\Delta \mathrm{H}_{\mathrm{m}} 100 \%\right)^{1}$ as shown in Equation 2.

$$
\chi=\frac{\Delta H_{m}}{\Delta H_{m} 100 \%}
$$

An estimate of the fusion heat of an infinite crystal of 3 -PHB was found to be $146 \mathrm{Jg}^{-1}$ which is the usual value used in this calculation ${ }^{1}$.

From this approach (taken from the second heating of DSC), the values found for crystallinity of PHB thermo-compression molded film and nanofibers mat were $53 \%$ and $55 \%$ respectively. With such results, one might suggest that the type of processing did not affect the PHB crystallinity, unlike what has been indicated by using FTIR analysis (Tables 2 and 3). However, this result disagrees with other results found in the literature. When as-received PHB powder was compared with PHB-based fibrous material, the latter displayed values of melting temperature and enthalpy slightly higher than those corresponding to the powder precursor $^{21}$. Such result was attributed to the orientation of
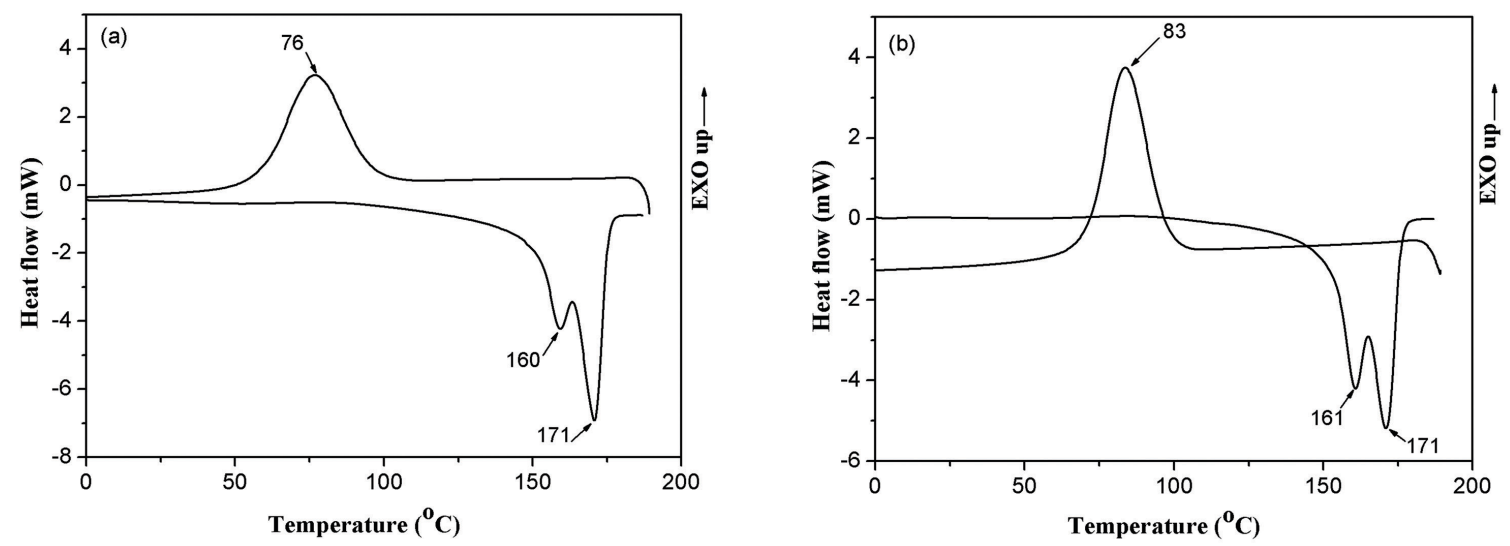

Figure 4. Cooling and second heating curves obtained by DSC of: (a) PHB thermo-compression molded film and (b) PHB electrospun nanofibers mat. 
macromolecular chains in the longitudinal fiber direction during the electrospinning process, which may have promoted crystallization.

Regarding the results found in the DSC study, one possible explanation for the apparent discrepancy with the previous results was given by $\mathrm{Xu}$ et al. ${ }^{9}$. According to these authors, the crystallinity calculated from the endotherm may not be the original one due to the further crystallization, which frequently occurs in polyhydroxyalkanoates caused by the relatively slow crystallization rate.

Small-angle X-ray scattering (SAXS) is a well-established technique for probing the morphology of polymers and provides information describing structural features of the order of 10-1000 $\AA$ in size.

Figure 5 illustrates the SAXS 2D patterns of the PHB film and the nanofibers mat.

Isotropic ring patterns are observed in Figure $5 \mathrm{~b}$ due to the nonwoven membrane presence ${ }^{22}$. The same ring pattern has already been observed in a light scattering from membranes and was attributed to the morphology regularity ${ }^{23}$.

In samples possessing a regular, ordered morphology, a correlation peak is observed in the SAXS intensity at a characteristic value of $\mathrm{q}$ (denoted by $\mathrm{q}_{\max }$ ), which is related to the domains separation or heterogeneities in the material ${ }^{24}$. In the simplest analysis, the scattering may be treated according to Bragg's Law and hence:

$q_{\max }=\frac{2 \pi}{d}$

Where $d$ is the average inter-domain spacing and $q$ is the scattering vector defined as follow:

$q=\frac{4 \pi}{\lambda} \sin \theta$

Where $\lambda$ is the X-ray wavelength and $\theta$ is the scattering angle.

In the case of a semicrystalline polymer possessing a lamellar morphology (such as PHB and PHB-HV), which is isotropically distributed throughout the material, the Lorentz correction is applied to the SAXS intensity prior to the peak position determination ${ }^{11}$.

The Lorentz correction involves the intensity function multiplication by a factor of $\mathrm{q}^{2}$ and has the effect of shifting the position of a broad correlation peak to higher values of q. The Lorentz corrected SAXS profiles, constructed by plotting I (q) $\mathrm{q}^{2}$ versus $\mathrm{q}$, for PHB film and PHB nanofibers mat are displayed in Figure 6.

At a first glance the higher intensity of the scattering vector presented in Figure 6b suggests that PHB nanofibers mat has larger crystalline lamellae thickness and PHB film has higher amorphous fraction.

According to Rule, Liggat ${ }^{25}$, a simple, ideal lamellar model is used to describe certain semicrystalline polymers. The d-spacing represents the distance between adjacent crystalline lamellae which are separated by amorphous domains and arranged in space-filling stacks (Figure 7).

The d-spacing is thus equivalent to the sum of the crystalline and amorphous layer thicknesses $\left(1_{c}\right.$ and $l_{a}$ respectively):

$d=l_{c}+l_{a}$

An independent knowledge of the sample crystallinity degree $(\chi)$ enables both of the above parameters to be estimated, assuming the lamellar stacks to be space filling ${ }^{25}$ :

$$
\begin{aligned}
& l_{c}=\chi d \\
& l_{a}=(1-\chi) d
\end{aligned}
$$

The d-spacing can be estimated from Eq. 3 with $\mathrm{q}_{\max }$ corresponding to the maximum of I (q) $q^{2}$ versus $q$ curves $^{26}$.

Scattering peaks were hardly detected for PHB film (Figure 6a) and PHB nanofibers mat (Figure 6b) denoting a low electron density contrast between the crystalline and amorphous phases. This difficulty had already been observed previously for polypropylene fibers obtained by electrospinning. A scattering peak was evidently detected for the aligned fibers, whereas a small shoulder or no peak was observed for the random fibers ${ }^{22}$. (a)

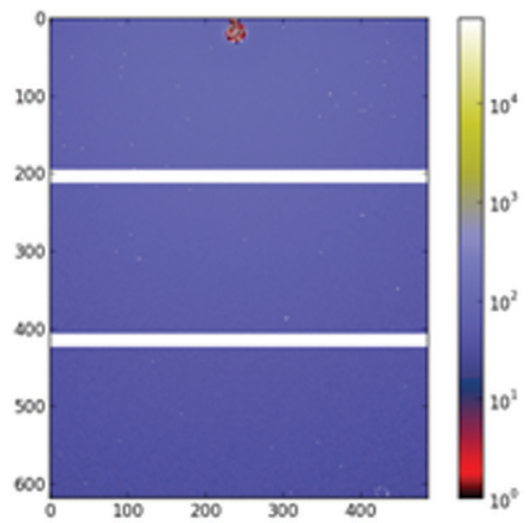

(b)

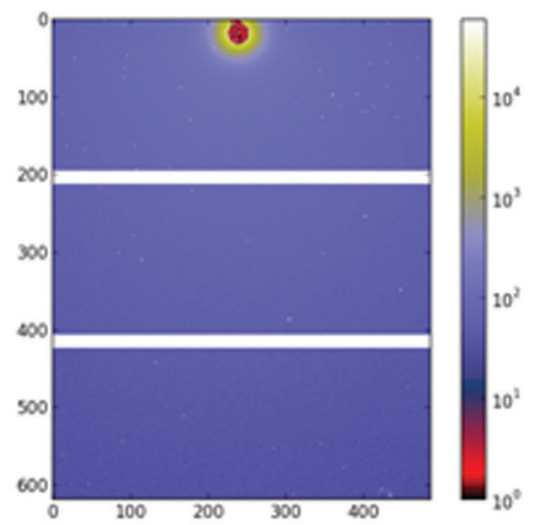

Figure 5. SAXS 2D patterns of: (a) PHB thermo-compression molded film and (b) PHB nanofibers mat. 
In the present study, we have roughly identified $\mathrm{q}_{\max }$ values of 0.26 and $1.43 \mathrm{~nm}$ for PHB nanofibers and $1.39 \mathrm{~nm}$ for PHB film. The estimated values of $1_{c}$ and $l_{a}$ by applying the above equations, and using the values of crystallinity degree $(\chi)$ found by DSC are displayed in Table 4.

Due to the difficulty to identify $\mathrm{q}_{\max }$ on the Figure 6 , the values presented in Table 4 serve as relative values in order to compare both morphologies of PHB, film and nanofibers.

Values established for $\mathrm{l}_{\mathrm{c}}$ and $\mathrm{l}_{\mathrm{a}}$ found in the literature for PHB films were $12-47^{\mathrm{c}} \AA$ and $8-14 \AA$ respectively ${ }^{16}$; $5.79-8.87 \mathrm{~nm}$ and $2.76-3.75 \mathrm{~nm}$ respectively ${ }^{27} ; 28.5 \AA$ and $26.9 \AA$ respectivel $y^{28}$. As can be noticed, the lamellar crystals thickness was always larger than that of the amorphous layers, as we have also found.

Structural characterization based on WAXS and SAXS analyses performed by Wang et al., ${ }^{29}$ revealed that polyethylene

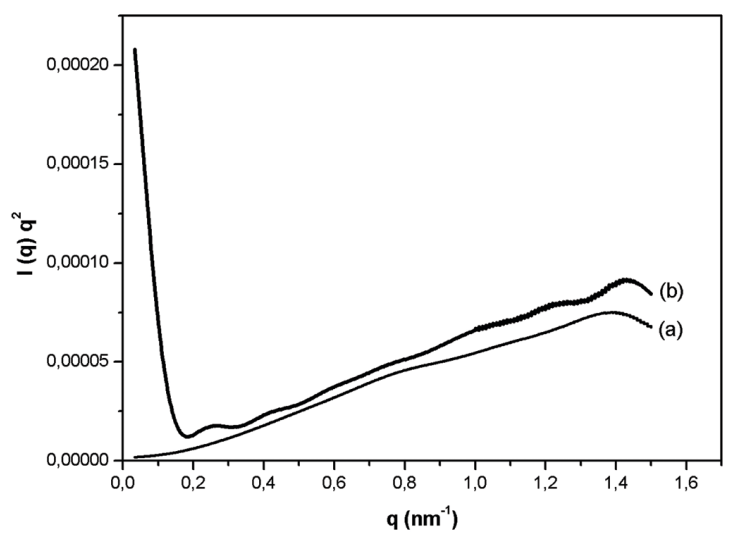

Figure 6. Lorentz corrected SAXS profiles of: (a) PHB thermocompression molded film and (b) PHB nanofibers mat.

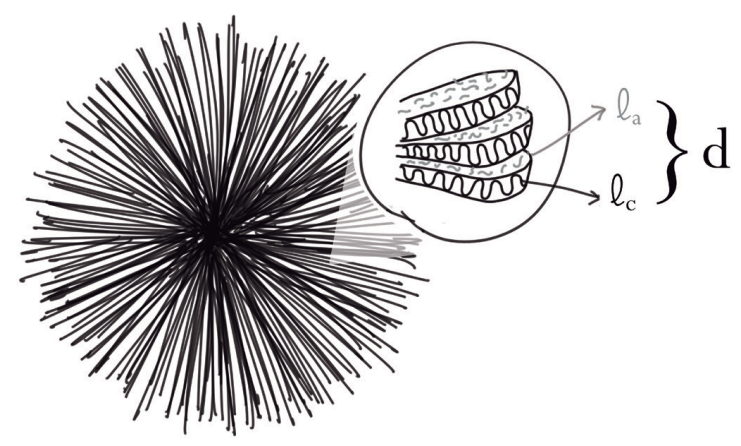

Figure 7. Ideal lamellar model showing d-spacing between adjacent crystalline lamellae.

Table 4. Morphological parameters of the lamellar structure of PHB thermo-compression molded film and PHB nanofibers mat.

\begin{tabular}{lccc}
\hline \multicolumn{1}{c}{ Sample } & $\mathbf{l}_{\mathbf{c}}(\boldsymbol{\AA})$ & $\mathbf{l}_{\mathbf{a}}(\boldsymbol{\AA})$ & $\mathbf{d}(\boldsymbol{\AA})$ \\
\hline Film & 24 & 21 & 45 \\
Nanofibers & 133 & 108 & 241 \\
& 24 & 20 & 44 \\
\hline
\end{tabular}

$\chi$ values obtained from DSC are 0.53 and 0.55 for the film and nanofibers respectively. terephthalate (PET) as spun fibers consisted mainly of two phases, namely, amorphous and oriented non-crystalline phase $(\mathrm{ON})$, with a trace of crystalline phase. According to the authors, such ON phase is like located outside the lamellar stacks, giving rise to an insignificant contribution to the scattering pattern. As reported by them, further heating to higher temperatures led to the gradual transformation of the ON phase to crystalline phase. It is worth to mention that ageing produces the same effect in PHB, that is, crystal perfection over time ${ }^{28}$.

Herein the fibers did not suffer annealing neither much time has passed until characterization, so probably there is still a large fraction of ON phase in the PHB nanofibers mat which giving rise to the ring pattern observed in Figure 5. In relation to PHB film, the secondary crystals formation, as pointed by $\mathrm{XRD}$, has a small contribution to $\mathrm{l}_{\mathrm{c}}^{27}$.

In poly(vinylidene fluoride) (PVDF)/PHB blends produced by casting neat PVDF showed a stronger scattering peak than neat PHB. This was attributed to the higher electron density contrast between the alternating crystalline and amorphous layers of PVDF $\left(\Delta \eta=0.103 \mathrm{~mol} \mathrm{~cm}^{-3}\right)$ compared to that of $\operatorname{PHB}\left(\Delta \eta=0.050 \mathrm{~mol} \mathrm{~cm}^{-3}\right)^{30}$. It was mentioned a time period over which PHB was a "temporary amorphous diluent" with respect to PVDF with an extra lamellar segregation of this temporary amorphous diluent $t^{30}$.

Corroborating with the aforementioned temporary amorphous phase, the two melting peaks observed in the DSC experiments (Figure 4) had been explained by the presence of an amorphous-crystalline lamellar interface in as-processed $\mathrm{PHB}^{28}$. According to the proposed theory, this interface, which is still not constrained by the perfection process that characterizes ageing, is susceptible to suffer rearrangement. This rearrangement is reflected in a shift of the melting peak toward higher temperatures indicating the crystalline lamellae thickening ${ }^{28}$.

In this context we might suggest that there was not enough scattering contrast in both samples in the SAXS experiments and the values shown in Table 4 are not reliable as absolute values.

The PHB thermal stability in the different forms was then investigated by TGA (Figure 8).

Thermal degradation of pure PHB occurs mainly through one step, which is characterized by a single peak on DTG curves as we found here ${ }^{18}$.

As can be noted on Figure 8, the maximum mass loss rate for PHB film occurred at $285{ }^{\circ} \mathrm{C}$, while PHB-based fibrous material displayed lesser thermal stability with maximum mass loss rate at $262{ }^{\circ} \mathrm{C}$. The values found are consistent with those found by Ramier et al. ${ }^{21}$ and could be attributed to the higher specific surface area of the fibrous structures which was more affected by heating. According to the aforecited authors, as the heating spread by conduction within the materials, the fibers characterized by a large surface area could reach the degradation temperature faster. Hence, if the surface area seems to be the predominant factor in thermal stabilization, the results presented by TGA were inconclusive with respect to correlate the thermal stability with the two forms of PHB crystallinity. 
A comparison (PHB thermo-compression molded film versus nanofibers mat) of the mechanical properties in tensile mode is shown in Figure 9.

The values related to PHB thermo-compression molded film were not in agreement with those found in the literature, especially the value of the tensile modulus (Figure 9c). PHB films are known to have fragile behavior with an elongation at break below $5 \%$ and an elastic modulus around $1.5 \mathrm{GPa}^{5}$. This was attributed to the relatively high crystallinity of $\mathrm{PHB}$ homopolymer, and in particular the significant secondary crystallization that occurs post processing with age ${ }^{1}$ or by the temperature action ${ }^{13}$. According to Kurusu et al. ${ }^{28}$ it is important to know how much time has passed after the melt processing before analyzing the PHB samples. These authors controlled the post processing time and found that after 14 days the elastic modulus shifted from 0.6 to $1.5 \mathrm{GPa}$. In addition, the PHB mechanical properties are known to be dependent on the polymer composition and processing conditions, and in particular on whether or not the polymers were produced using solvent casting or melt mixing and casting ${ }^{1}$. Domínguéz-Díaz et al. ${ }^{31}$ reported elastic modulus in the range of $850 \mathrm{MPa}$ for as-cast PHB film prepared dissolving the polymer in chloroform and with the same molecular weight used by us.

Another example of how the processing conditions have influenced on mechanical properties had been given by Kurusu et al. ${ }^{28}$. According to such authors, in contrast to what was already reported, annealing had a negligible or negative effect on the mechanical properties of their compositions tested, including pure $\mathrm{PHB}$ processed by extrusion, mixing and injection molding.

Herein, the values of tensile strength and tensile modulus for the PHB nanofibers mat are considerably lower than those of PHB film. This is in accordance with the literature. Fernandes et al. ${ }^{5}$, just as we did, observed that PHB nanofibers mats were fragile and they easily broke during clamping. As explained by them, since the mats are composed of many voids, is comprehensible that they are less resistant, obviously taking into account the thickness of the mats. Moreover, PHB mat in the present study was prepared from PHB dissolved in chloroform and DMF, producing a material with lower mechanical properties ${ }^{31}$.
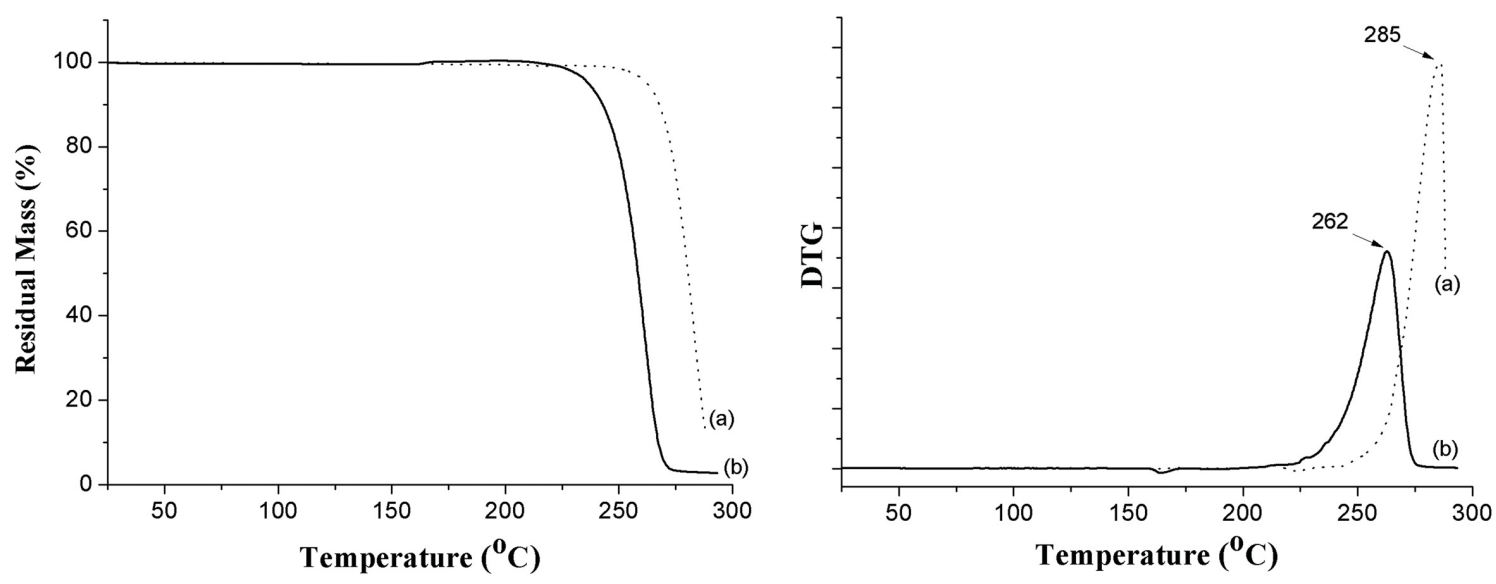

Figure 8. TG and DTG curves of: (a) PHB thermo-compression molded film and (b) PHB nanofibers mat.
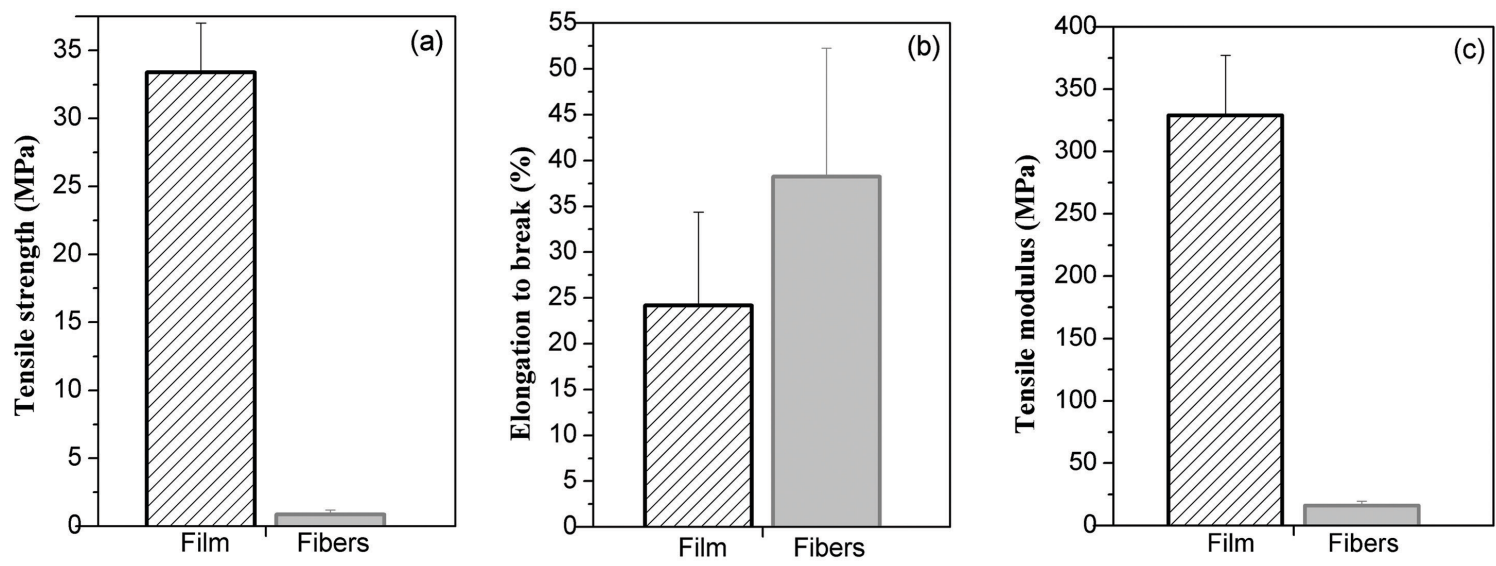

Figure 9. Tensile properties of PHB thermo-compression molded film and nanofibers mat: (a) tensile strength, (b) elongation to break and (c) tensile modules. 

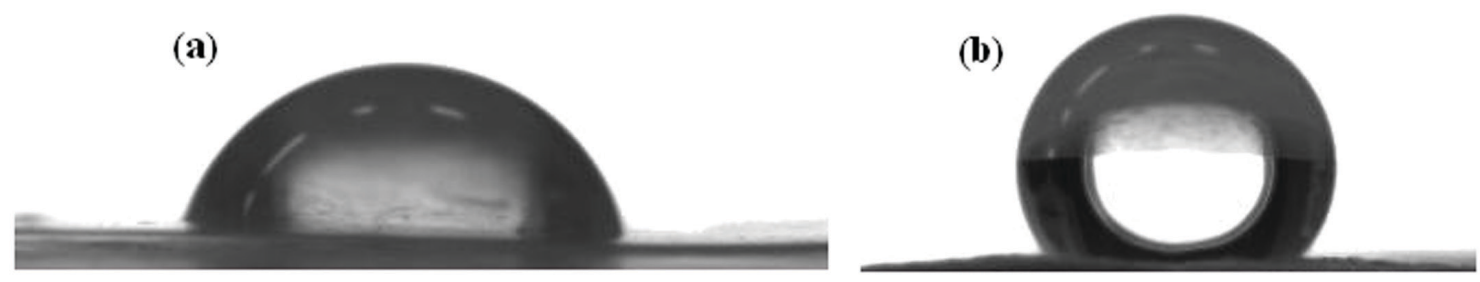

Figure 10. Shape of water drop on the air side of: (a) PHB film $(\theta=77.10 Æ)$ and (b) PHB nanofibers mat $(\theta=130.30 Æ)$.

The crystallinity degree alone is effective in determining the stiffness and yield point for most crystalline polymers, and these properties are independent of molecular weight ${ }^{8}$. As a result, they can be expressed as single-valued functions of crystallinity degree. As crystallinity decreases, both stiffness and yield stress decrease ${ }^{8}$. Hence, according to the bar charts shown in Figure 9, one possible suggestion is that, at the experiment conditions, PHB film behaviors as more crystalline than PHB nanofibers mat. This suggestion is consistent with the results of FTIR and WAXD but goes against the findings of DSC. In this regard, we might propose that PHB nanofibers mat in fact has low crystallinity degree with a metastable amorphous state ${ }^{8}$. The raise of temperature during DSC experiment might led to the gradual transformation of the metastable amorphous phase to crystalline phase and a high value of crystallinity degree was achieved $(\chi=55 \%)$.

It is worth remembering that the values of $l_{c}$ and $l_{a}$ (Table 4) found by SAXS were based on $\chi$ obtained by DSC, which were very close for the film and nanofibers mat. Hence, one might suppose is that the greater values of $133 \AA$ and $108 \AA$ of $1_{c}$ and $l_{a}$ respectively found for the fibers (Table 4) are related to the phase change occurred during the DSC experiment.

Hydrophilic / hydrophobic properties are of major importance in specific applications. Therefore, the samples were also examined for their contact angle (Figure 10).

As can be seen in Figure 10, PHB nanofiber mat presents hydrophobic properties. Fryczkowski et al. ${ }^{32}$ found contact angle just below 90 degrees for both PHB electrospun fibers derived from 2,2,2-trifluoroethanol (TFE) or chloroform solutions. On the other hand, Yang et al..$^{33}$ reported contact angle of the poly(3-hydroxybutyrate-co-3-hydroxyvalerate) (PHBV) cast film and PHBV electrospun film with same concentration of $4 \%$ in chloroform of $88^{\circ}$ and $130^{\circ}$ respectively.

\section{References}

1. Laycock B, Halley P, Pratt S, Werker A and Lant P. The chemomechanical properties of microbial polyhydroxyalkanoates. Progress in Polymer Science. 2014; 39(3-4):536-583.

2. Bucci DZ, Tavares LB and Sell I. Biodegradation and physical evaluation of PHB packaging. Polymer Testing. 2007; 26:908915.

3. Gredes T, Gedrange T, Hinüber C, Gelinsky M and KunertKeil C. Histological and molecular -biological analyses of poly (3-hydroxybuyrate) (PHB) patches for enhancement of bone regeneration. Annals of Anatomy-Anatomisher Anzeiger. 2015;199:36-42.
According to them, PHBV electrospun film had a much higher contact angle due to its surface roughness. Ramier et al. ${ }^{21}$ reported contact angle for PHB mat on the air side and on the collector side of $120 \pm 4^{\circ}$ and $103 \pm 3^{\circ}$ respectively. According to these authors, the decrease in contact angles for the fiber surface touching the collector could be related to a decrease in specific surface areas of the first fiber layer that flattened in contact with the aluminum collector. An average contact angle equal to $80 \pm 2^{\circ}$ for PHB film was reported by the same authors. In general the values of contact angle found by us are in accordance with those of the literature and might be ascribed to the higher (fibers) or lower (film) specific surface area.

\section{Conclusion}

PHB electrospun nanofibers mat was compared to PHB thermo-compression molded film, mainly in terms of crystallinity. FTIR and WAXD indicated that PHB thermo-compression moulding produces PHB film with higher crystalline degree. On the other hand, when PHB is electrospun initially its crystallinity degree is lower, but as suggested by DSC experiment, a metastable amorphous phase gradual transformation to crystalline phase occurs when temperature rises.

As a conclusion, this study indicated that when dealing with PHB, the processing conditions as well as the analyses conditions are capable of affecting the crystallinity degree and hence PHB properties.

\section{Acknowledgements}

The authors acknowledge the financial support from Coordination of Improvement of Senior Staff (CAPES), CNPq, Fapemig and LNLS.

4. Porter MM and Yu J. Crystallization kinetics of poly(3Hydroxybutyrate) granules in different environmental conditions. Journal of Biomaterials and Nanobiotechnology. 2011; 2:301310. http://dx.doi.org/10.4236/jbnb.2011.23037.

5. Fernandes JG, Correia DM, Botelho G, Padrão J, Dourado F, Ribeiro C, et al. PHB-PEO electrospun fiber membranes containing chlorhexidine for drug delivery applications. Polymer Testing. 2014; 34:64-71.

6. Veleirinho B, Ribeiro-do-Valle RM and Lopes-da-Silva JA. Processing conditions and characterization of novel electrospun poly (3-hydroxybutyrate-co-hydroxyvalerate)/chitosan blend fibers. Materials Letters. 2011; 65(14):2216-2219. http://dx.doi. org/10.1016/j.matlet.2011.04.058. 
7. Nagiah N, Madhavi L, Anitha R, Anandan C, Srinivasan NT and Sivagnanam UT. Development and characterization of coaxially electrospun gelatin coated poly (3-hydroxybutyric acid) thin films as potential scaffolds for skin regeneration. Materials Science and Engineering C. 2013; 33(7):4444-4452.

8. Billmeyer FW Jr. Textbook of polymer Science. 2nd ed. New York: John Wiley and Sons; 1971. p. 110, 216, 235.

9. Xu J, Guo BH, Yang R, Wu Q, Chen GQ and Zhang QM. In situ FTIR study on melting and crystallization of polyhydroxyalkanoates. Polymer. 2002; 43:6893-6899.

10. Zhang J, Sato H, Noda I and Ozaki Y. Conformation rearrangement and molecular dynamics of poly(3-hydroxybutyrate) during the melt-crystallization process investigated by infrared and twodimensional infrared correlation spectroscopy. Macromolecules. $2005 ; 38: 4274-4281$.

11. Wang C, Hsu CH and Hwang IH. Scaling laws and internal structure for characterizing electrospun poly[(R)-3-hydroxybutyrate] fibers. Polymer. 2008; 49(19):4188-4195.

12. Pachekoski WM, Dalmolin C and Agnelli JAM. The influence of the industrial processing on the degradation of poly(hidroxybutyrate) - PHB. Materials Research. 2013; 16(2):327-332.

13. Zhijiang $\mathrm{C}$, Chengwei $\mathrm{H}$ and Guang Y. Crystallization behavior, thermal property and biodegradation of poly(3-hydroxybutyrate)/ poly(ethylene glycol) grafting copolymer. Polymer Degradation \& Stability. 2011; 96:1602-1609.

14. Sun X, Guo L, Sato H, Ozaki Y, Yan S and Takahashi I. A study on the crystallization behavior of poly ( $\beta$-hydroxybutyrate) thin films on si wafers. Polymer. 2011; 52:3865-3870.

15. Pinto CE, Arizaga GG, Wypych F, Ramos LF and Satyanarayana KG. Studies of the effect of molding pressure and incorporation of sugarcane bagasse fibers on the structure and properties of poly (hydroxy butyrate. Composites. Part A, Applied Science and Manufacturing. 2009; 40:573-582.

16. Canetti M, Urso M and Sadocco P. Influence of the morphology and of the supermolecular structure on the enzymatic degradation of bacterial poly(3-hydroxybutyrate). Polymer. 1999; 40(10):25872594.

17. Fabri D, Guan J and Cesàro A. Crystallisation and melting behaviour of poly (3-hydroxybutyrate) in dilute solution: towards an understanding of physical gels. Thermochimica Acta. 1998; 321(1-2):3-16.

18. Erceg M, Kovcic T and Klavic I. Thermal degradation of poly(3-hydroxybutyrate) plasticized with acetyl tributyl citrate. Polymer Degradation \& Stability. 2005; 90:313-318.

19. Righetti MC and Di Lorenzo ML. Melting temperature evolution of non-reorganized crystals. Poly(3-hydroxybutyrate). Thermochimica Acta. 2011; 512(1):59-66. http://dx.doi. org/10.1016/j.tca.2010.08.023.

20. Bezerra EB, Leite AM, Araújo EM and Mélo TJ. Obtenção e caracterização de membranas obtidas a partir de blendas poliméricas de poliamida. Polímeros. 2014; 24(3):381-387.

21. Ramier J, Bouderlique T, Stoilova O, Manolova N, Rashkov I, Langlois V, et al. Biocomposite scaffolds based on electrospun poly(3-hydroxybutyrate) nanofibers and electrosprayed hydroxyapatite nanoparticles for bone tissue engineering applications. Materials Science and Engineering C. 2014; 38:161-169.

22. Jao CS, Wang Y and Wang C. Novel elastic nanofibers of syndiotactic polypropylene obtained from electrospinning. European Polymer Journal. 2014; 54:181-189.

23. Umaña E, Ougizawa T and Inoue T. Preparation of new membranes by complex formation of itaconic acid-acrylamide copolymer with polyvinylpyrrolidone: studies on gelation mechanism by light scattering. Journal of Membrane Science. 1999; 157:8596.

24. Chang SL, Yu TL, Huang CC, Chen WC, Linilu K and Lin TL. Effect of polyester side-chains on the phase segregation of polyurethanes using small-angle X-ray scattering. Polymer. 1998; 39(15):3479-3489.

25. Rule RJ and Liggat JJ. Time-resolved synchrotron small angle X-ray scattering studies of poly(3-hydroxybutyrate) and poly(3hydroxybutyrate-co-3-hydroxyvalerate) polymers. Polymer. 1995; 36(20):3831-3840.

26. Patrício PS, Pereira IM, Silva NC, Ayres E, Pereira FV and Oréfice RL. Tailoring the morphology and properties of waterborne polyurethanes by the procedure of cellulose naocrystal incorporation (5658). European Polymer Journal. 2013; 49(12):3761-3769.

27. Heo K, Yoon J, Jin KS, Kim G, Sato H, Ozaki Y, et al. Synchrotron $\mathrm{X}$-ray scattering studies on the structural evolution of microbial poly(3-hydroxybutyrate). Journal of Applied Crystallography. 2007; 40:s594-s598.

28. Kurusu RS, Siliki CA, David É, Demarquette NR, Gauthier C, Chenal JM. Incorporation of Plasticizers in sugarcane-based poly(3-hydrixybutyrate) (PHB): Changes in microstructure and properties through ageing and annealing. Industrial Crops and Products. 2015; 72:166-174.

29. Wang C, Lee MF and Jao CH. Phase transition of poly(ethylene terephthalate) in nanofibers electrospun from phenol-based solution. European Polymer Journal. 2014; 52:127-136.

30. Chiu HJ, Chen HL and Lin JS. Crystallization induced microstructure of crystalline/crystalline poly(vinylidenefluoride)/ poly(3-hydroxybutyrate) blends probed by small angle X-ray scattering. Polymer. 2001; 42(13):5749-5754.

31. Domínguez-Díaz M, Meneses-Acosta A, Romo-Uribe A, Peña C, Segura D and Espin G. Thermo-mechanical properties, microstructure and biocompatibility in poly- $\beta$-hydroxybutyrates (PHB) produced by OP and OPN strains of Azotobacter vinelandii. European Polymer Journal. 2015; 63:101-112.

32. Fryczkowski R, Gorczowska M, Fryczkowska B and Janicki J. The effect of solvent on the properties of nanofibers obtained by electrospinning from a mixture of poly(3-hydroxybutyrate) and polyaniline. Synthetic Metals. 2013; 166:14-21.

33. Yang D, Zhang J, Xue J, Nie J and Zhang Z. Electrospinning of Poly(3-hydroxybutyrate-co-3-hydroxyvalerate) nanofibers with feature surface microstructure. Journal of Applied Polymer Science. 2012; 127(4):2867-2874. 